\title{
ECHOGRAM IN MELANOMA OF THE CHOROID*
}

\author{
BY \\ ARVO OKSALA \\ From the Ophthalmic Department of the Central Finland Regional Hospital, Jyväskylä, Finland
}

DiAGNOSIS is often difficult in melanoma of the choroid. This is evidenced e.g. by frequent misdiagnosings which come to light only after the enucleation of the bulbus (Reese, 1957) and by numerous more or less reliable methods of examination which have been applied for the determination of this tumour. Among the latest and, judging by the initial results, the most reliable methods of examination is the diagnostic use of the echogram.

Mundt and Hughes (1956) and Oksala and Lehtinen (1957) were the first to use ultrasound in the diagnosis of intrabulbar tumours. These investigators found that, even in those cases in which ophthalmoscopic exploration of the tumour was prevented by detachment of the retina or opacity of the intermediary substances of the eye, it was possible to diagnose the intrabulbar tumour with a high degree of certainty by means of echoes registered by an ultrasonic apparatus. Oksala and Lehtinen came to the conclusion that the echoes reflected from a choroidal melanoma could be positively observed on the screen of the Braun tube even at the stage when the height of the tumour was only about $2-3 \mathrm{~mm}$. On the basis of these observations the echogram traced by a melanoma of the choroid was marked by the typical feature that the tumour tissue produced on the screen an irregular accumulation of echoes, the echogram varying somewhat according to the direction of investigation.

The line traced by the echo and visualized on the screen did not return to the zero-line after having encountered the tumour. This finding was based on the echogram obtained from one patient with melanoma of the choroid. In this case the tumour was fairly small, its height being $4-5 \mathrm{~mm}$.

If the intrabulbar tumour is larger, we shall of course obtain on the screen a longer echogram which is more easily examined. In the first patient now reported by me the choroidal melanoma could not be diagnosed before the tumour had grown to a height of $10 \mathrm{~mm}$. A disease of this kind provided a first-rate opportunity of studying the echogram given off by melanoma of the choroid. Since the literature dealing with this new method of research is very scanty, and since it is by no means always easy to interpret echograms produced by various diseases of the eye, a report of two cases together with the results of investigation appears justified.

* Received for publication September 15, 1958. 


\section{Case Reports}

Case 1, a man aged 52 with previously good health, had noticed about 7 months before coming for a consultation a slight shadow in the upper part of the right visual field. The shadow grew very slowly. Since the patient did not feel any pain and the eye had a sound appearance, he thought he would wait for the shadow to disappear of itself. However, the spreading of the shadow and a sudden deterioration of visual acuity finally brought him to our Ophthalmic Department.

Ophthalmological Examination (11.4.58).-Visual acuity was hand movements in the right eye and 1.0 in the left. The anterior parts of both eyes were symptom-free and the intermediary substances clear. Gonioscopic examination did not reveal any pathological changes. Intra-ocular pressure in both eyes was $18 \mathrm{~mm} . / \mathrm{Hg}$ (Schiötz). The visual field of the right eye showed in its upper part an extensive absolute defect which reached as far as the central area of the visual field. With ophthalmoscopy one could see low down in the right fundus extensive detachment of the retina extending to the macula and the optic disc. In the middle of the detachment the retina appeared so compact and dark that an intrabulbar tumour, primarily a choroidal melanoma, suggested itself. Diascleral examination also gave indications of a tumour. Elsewhere the retina seemed normal. The left fundus showed no pathological changes.

General examination of the patient yielded no pathological features. Sedimentation rate $8 \mathrm{~mm}$. $/ \mathrm{hr}$. The blood count was normal. The Wassermann reaction and Kahn and Sitolipin tests were negative. Roentgenological examination of the thorax and cranium was non-contributory.

Examination by Ultrasound Equipment (14.4.58).-Since echograms of the eye registered by ultrasonic apparatus had been applied with success at our Ophthalmic Department for over 2 years in the diagnosis of intrabulbar tumours, such an investigation was performed in this case as well. The equipment employed was constructed by Drs. J. and $\mathbf{H}$. Krautkrämer, Gesellschaft für Elektrophysik, Köln (Fig. 1). The functioning of this apparatus has been described by Oksala and Lehtinen (1957). A searching unit was used in this investigation, which had been constructed by the manufacturers mentioned on the basis of the experience collected at our Ophthalmic Department, particularly for diagnosing diseases of the eye. At the bottom of Fig. 1 a barium titanate searching unit is seen; the unit had a diameter of $5 \mathrm{~mm}$., and a frequency of $6 \mathrm{Mhz}$, and was plated with silver.

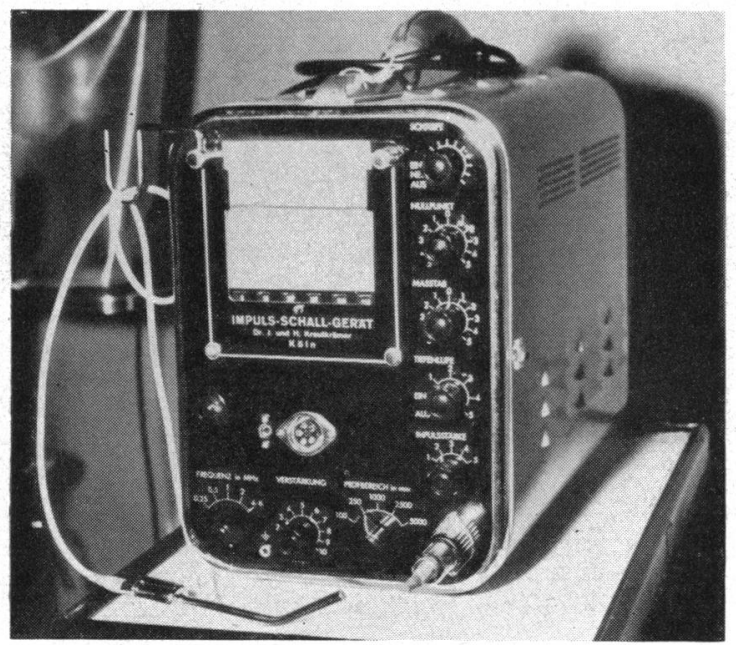

FIG. 1.-Ultrasonic equipment.

Below at the end of the cable is a searching unit of $5 \mathrm{~mm}$. diameter and $6 \mathrm{Mhz}$, which has been specially constructed for ophthalmological examinations. At the upper part of the apparatus is seen the screen of the Braun tube, on which the echogram is shown. 
The eye was anaesthetized for examination by 3.0 per cent. Cornecain drops (Hoechst), and the blepharostat was put into position. As sound transmitter, simultaneously forming a protective film between the unit and the eye, we used a 1.0 per cent. methylcellulose solution. The searching unit was lightly pressed against the surface of the eye, and the sound was then directed against the fundus from different angles, by adjusting the position of the unit, the fundus thus being thoroughly investigated. The necessary photographs were taken of the echoes visualized on the screen of the Braun tube. The scale was adjusted during the investigation so that the echogram emitted by the tumour and visible on the screen became as long as possible.

In the right eye normal echoes were obtained everywhere from above. Fig. 2A is an example of such a normal echogram. The initial broad echo on the left is only partly seen on the photograph for lack of space. The initial echo is produced by both the apparatus itself and the anterior wall of the eye. The initial echo is followed by the zero-line typical of a normal vitreous, and a high and sharp tracing of the echo from the posterior wall of the eye is seen to the right. The echoes emitted by the anterior and posterior surface of the lens are visualized if the sound wave is directed axially. In Fig. $2 \mathrm{~A}$ the lens is completely passed by the sound.

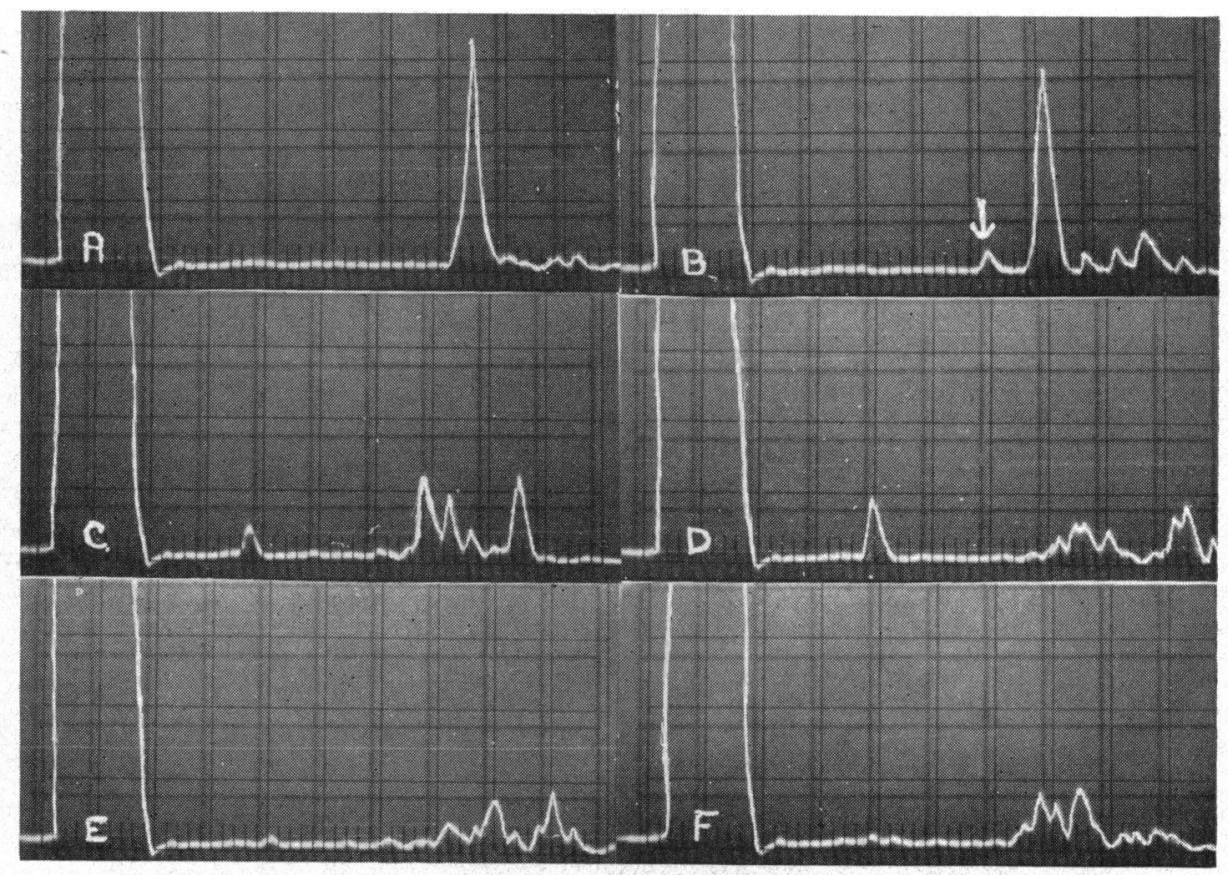

Fig. 2.-Typical echograms obtained from the first patient. "A" is an example of a normal echogram when the sound passes through the globe and by the lens. To the left a high initial echo is seen, which does not find sufficient space on the screen. The initial echo is followed by the zero-line, and to the right is the high peak produced by the posterior bulbar wall.

In " $B$ " the sound encounters the detached retina but not yet the tumour. The arrow marks the echo emitted by the detached retina. This echo is followed by a short zero-line, typical of the subretinal fluid.

In " $C$ ", "D", " $E$ ", and " $F$ " the sonic wave encounters the tumour tissue from different directions, and different echoes given off by the tumour tissue are traced.

In " $C$ ", and " $D$ " the low peak of the posterior wall of the lens is seen in the middle of the vitreous. 
When directing the sonic wave against the detachment of retina it could be observed that there was in the centre of the detachment behind the retina an acoustically very heterogeneous tissue, with a considerably higher density than that of the vitreous. The height of the tumour could be read on the scale as about $10 \mathrm{~mm}$., the width being about $5 \mathrm{~mm}$. The retina was adherent to the tumour over a short distance. Fig. 2B illustrates the echogram from the region of the detached retina, but from beside the tumour. The pathological change revealed here is the fairly low echo emitted by the detached retina (arrow) immediately in front of the echo given off by the posterior bulbar wall. Figs 2C and 2D show nearly in the middle of the vitreous the rather low echo from the posterior surface of the lens. Figs 2C, D, E, and F demonstrate on the right various echoes emitted by the tumour tissue. In each photograph the sound has traversed the tumour from a slightly different angle. In Fig. 2E the sound passes through the tumour almost longitudinally, on account of which the echogram produced by the tumour is the longest of all. In the points shown on Figs $2 \mathrm{C}, \mathrm{D}, \mathrm{E}$, and $\mathrm{F}$, the retina does not produce a separate echo, as it is adherent to the surface of the tumour.

The appended photographs have been selected from a considerable number and they illustrate typical echograms produced by melanoma of the choroid.

Treatment.-On April 17, 1958, enucleation of the right eye was carried out and the diagnosis of malignant melanoma of the choroid could be finally verified histologically.

Case 2, a man aged 45, who had previously had no diseases worth mentioning, had noted some 3 months before coming to the hospital a slowly growing shadow in the temporal part of the right eye. As visual acuity continued to deteriorate, he came to our Ophthalmic Department for examination.

Ophthalmological Examination (7.8.58). - Visual acuity $=0.4$ in the right and $=1.0$ in the left eye. The anterior parts of both eyes were asymptomatic and the intermediary substances clear. Nothing pathological was seen with gonioscopy. The ocular tension of both eyes was $20 \mathrm{~mm}$. $\mathrm{Hg}$ (Schiötz). The visual field of the right eye showed an extensive temporal defect, which extended to the optic disc and the macula. With the ophthalmoscope one could see nasally in the right fundus a high and circumscribed detachment of the retina, and the tissue below it gave a compact and richly pigmented appearance. Diascleral examination also supported the assumption of an intrabulbar tumour, probably melanoma of the choroid. Elsewhere the right fundus was normal. The left fundus revealed no pathological changes.

The patient was healthy, and the Wassermann reaction and Kahn and Sitolipin tests were negative. Roentgenological examination of the thorax and cranium did not bring to light any pathological features.

Examination by Ultrasound Equipment (8.8.58).-With a view to verifying the diagnosis of intrabulbar tumour, examination of the eye by ultrasound equipment was also undertaken. The apparatus and methods were identical with those described in the first case.

- Normal echograms were obtained from the right eye everywhere except in the area of retinal detachment. Fig. 3A (overleaf) shows a normal echogram, which was obtained when the sound wave was guided in the temporal direction. In Fig. 3B the sound has already encountered the margin of the tumour, and the short echo group produced by it is visible in front of the echo from the posterior bulbar wall. Figs $3 C, D, E$, and F show various echograms emitted by the tumour tissue. In no Figure is there any separate echo from the retina, which indicates, in conformity with the ophthalmoscopic findings, that the retina adhered to the tumour everywhere, or was at least very close to it. On the basis of the echograms the readings were about 5 to $6 \mathrm{~mm}$. for the height of the tumour and about $4 \mathrm{~mm}$. for its width. 


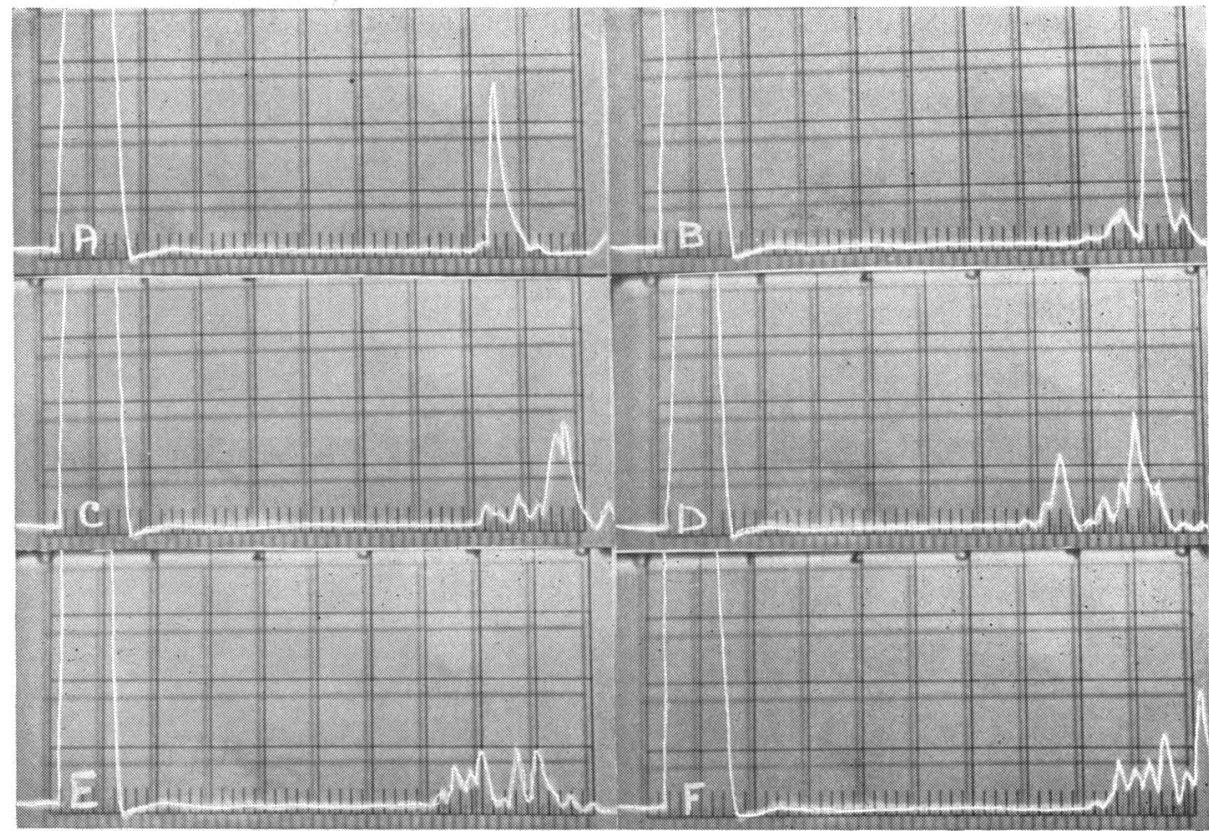

Fig. 3.- Echograms obtained from the second patient.

" $A$ " illustrates a normal echogram. In " $B$ " to " $F$ " the sound encounters the tumour tissue from different directions, and typical echograms indicating tumour tissue are obtained. The echo emitted by the posterior surface of the eye cannot easily be distinguished in " $D$ " to " $F$ ".

Treatment.-On August 10, 1958, enucleation of the right eye was performed. The clinical diagnosis of melanoma of the choroid could be further verified histologically.

\section{Discussion}

In the cases described above a fairly reliable diagnosis could be established clinically, with the aid of a diascleral examination, and with the echogram. In the first patient the ophthalmoscopic findings did not differ convincingly from those seen in idiopathic detachment of the retina. This case is an excellent example of the value of the echogram in the diagnosis of intrabulbar tumours. The echogram also enables the acoustic density of the subretinal space to be compared with that of the vitreous, so that the limits of a tumour mass, if present, can be assessed with an accuracy of 2 to $3 \mathrm{~mm}$. Such exact localization, independent of ophthalmoscopic exploration, may provide more favourable conditions for the $x$-ray and electrocoagulation treatment of intrabulbar tumours.

In studying the echograms obtained from a melanoma, it becomes apparent that the echogram changes with the direction of the investigation, the number and height of the echo peaks varying from one photograph to another. This is because the velocity of sound within the melanoma varies 
with the direction of investigation. Unlike the vitreous or the lens, the melanoma tissue is acoustically heterogeneous. This affects the formation of the individual echo and its height on the screen; it is thus possible to determine from which direction the ultrasonic wave encounters the limiting surface between two kinds of tissue with different velocities of sound, and also to estimate the degree of difference in velocity of sound between the two areas. The angle between the sound wave and these numerous limiting surfaces varies with great sensitivity following the movements of the searching unit and of the eye. At the site of the tumour the peak of the echo emitted by the posterior bulbar wall is lower than normal, because part of the ultrasound is absorbed by the tumour tissue, and because the variation between the velocity of sound in tumour tissue and the sclera is much slighter than the same variation between the vitreous and the sclera.

Although the melanoma echogram varies according to the direction of the sound, important common features can none the less be noted in different echograms. The echogram emitted by the melanoma is formed from numerous small echoes, and the echo only seldom, and then but for a moment, returns to the zero-line. Variations in velocity within the tumour tissue are so slight or the changes occur so slowly, that beyond the limiting surface of the tumour the peaks of individual echoes do not as a rule rise very high on the screen. However, if calcium conglomerations are present in the tumour tissue, their border surfaces will probably emit high echoes. This sensitive variation in the echogram according to the direction of the wave is in itself suggestive of the presence of tumour tissue.

The question of distinguishing between benign and malignant tumours, or between different kinds of tumour, still remains unsolved. In the author's opinion this is not yet possible with the available ultrasonic equipment.

\section{Differential Diagnosis}

From this viewpoint the most important condition is idiopathic detachment of retina. By way of differentiation from the melanoma echogram, this disease produces after the echo emitted by the retina a uniform zero-line continuing up to the echo from the posterior surface of the eye. Yet it should be borne in mind that, if the height of the melanoma is $<2 \mathrm{~mm}$., the echo it emits will fuse with that of the posterior bulbar surface.

In detachment of the choroid we can also determine by the echogram whether the wave has been reflected by a tumour or not. In postoperative detachment of the choroid, the subchoroidal space emits only a uniform zero-line like that of the vitreous and the subretinal fluid (Oksala, 1958).

In chronic uveitis the vitreous opacities may be so dense that they emit 
echoes. These echoes are, however, very low and usually much more sparse than those emitted by a melanoma (Fig. 4, arrows).

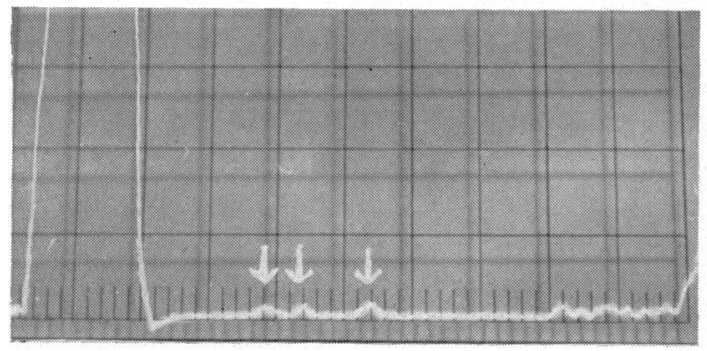

FIG. 4.-Echogram obtained from a patient with chronic uveitis, showing in the places marked by arrows the low echoes produced by dense opacities of the vitreous.

Connective tissue which has penetrated into the vitreous, e.g. in proliferating retinitis or because of trauma, also gives off echoes which can be seen on the screen, their height being approximately equal to that of echoes obtained from tumour tissue, but the individual echoes are again much sparser than those produced by a melanoma.

A less frequent problem of differential diagnosis is luxation of the lens. I once had to decide on the basis of the echogram whether tumour tissue or luxation had produced an absolute glaucoma. The patient had such an opaque cornea that even the slit lamp failed to reveal whether the lens was in place or not. In the echogram of luxation, one sees, about 4-5 $\mathrm{mm}$. in front of the reflection from the posterior surface of the eye, a fairly high echo from the anterior surface of the lens, and also a lower echo reflected by the posterior wall of the lens quite close to that from the posterior surface of the eye. Between these two echoes from the lens a uniform zero-line is visible. The diagnosis of luxation is further supported by the failure to obtain normal echoes from the lens.

\section{Summary}

The echogram registered by an ultrasonic apparatus has proved to be very useful in the diagnosis of intrabulbar tumours. The author has studied the echogram reflected by melanoma of the choroid in two cases. On account of the considerable height of the melanoma (10 and $6 \mathrm{~mm}$.), a fairly long echogram suitable for analysis was produced on the scale of the Braun tube in each case. It is usually possible to differentiate the echogram of a tumour from echograms traced in other types of intrabulbar disease.

\section{REFERENCES}

Mundt, G. H., and Hughes, W. F. (1956). Amer. J. Ophthal., 41, 488.

Oksala, A. (1958). Acta ophthal. (Kbh.), 36, 651.

- and LeHTINEN, A. (1957). Ophthalmologica (Basel), 134, 387.

ReEse, A. B. (1957). A.M.A. Arch. Ophthal., 58, 477. 